Décadrages Décadrages

cınéma, à travers champs Cinéma, à travers champs

15 | 2009

Raoul Ruiz

\title{
Le tableau vivant chez Raoul Ruiz : l'extension de la perception
}

\section{Valentine Robert}

\section{(2) OpenEdition}

\section{Journals}

Édition électronique

URL : http://journals.openedition.org/decadrages/88

DOI : $10.4000 /$ decadrages. 88

ISSN : 2297-5977

Éditeur

Association Décadrages

Édition imprimée

Date de publication : 1 octobre 2009

Pagination : 38-56

ISBN : 978-2-9700668-0-4

ISSN : 2235-7823

\section{Référence électronique}

Valentine Robert, "Le tableau vivant chez Raoul Ruiz : l'extension de la perception », Décadrages [En ligne], 15 | 2009, mis en ligne le 19 septembre 2014, consulté le 19 avril 2019. URL : http:// journals.openedition.org/decadrages/88 ; DOI : 10.4000/decadrages.88 


\section{Le tableau vivant chez Raoul Ruiz:}

\section{l'extension de la perception}

par Valentine Robert

1 L'étymologie du terme la plus précise est produite par Birgit Jooss, Lebende Bilder. Körperliche Nachahmung von Kunstwerken in der Goethezeit, Reimer, Berlin, 1999, pp. 19-22.

2 La recherche sur le tableau vivant, pratique méconnue et difficile à circonscrire puisque situé "entre" les disciplines théâtrales, artistiques et mondaines, est assez marginale. La première étude à "ressusciter " cet objet est signée Kirsten Gram Holmström, Monodrama, Attitudes, Tableaux vivants: Studies on Some Trends of Theatrical Fashion 17701815, Almquist \& Wiksell, Stockholm, 1967. C'est seulement en 2002 que les découvertes de Holmström sont relayées dans le champ francophone grâce à l'ouvrage de Bernard Vouilloux, Le Tableau vivant. Phryné, l'orateur et le peintre, Flammarion, Paris, 2002. S'il ne tient pas compte des approfondissements historiques remarquables qu'ont apportés Jack W. McCullough (dans Living Picture on the New York Stage, UMI Research press, Michigan, 1981) et surtout Birgit Jooss (op. cit.), l'ouvrage de Vouilloux fait émerger la notion de tableau vivant comme une référence dans le champ dit de l'esthétique, où elle connaît de nouveaux développements.

3 Nombre d'autres cinéastes, à l'instar de Pasolini ou Greenaway (avec lequel le directeur de la photographie de L'Hypothèse du tableau volé collaborera), ont exploré le motif, qui est par ailleurs incontournable dans la photographie contemporaine, très présent dans les performances et le cinéma expérimental, marquant également dans l'art chorégraphique, la publicité, et qui demeure même une pratique socioculturelle populaire dans les rues ou dans les fêtes telles que le festival "Pageant of the Masters" en Californie ou "Les Tableaux Vivants" au Québec.
Le cinéma ruizien est parcouru par un motif majeur: le tableau vivant. Accordons-nous d'abord sur une définition de cette notion. Le tableau vivant constitue une expression consacrée depuis le XVIII ${ }^{e}$ siècle ${ }^{\mathbf{1}}$, désignant une pratique dont l'origine remonte aux Passions liturgiques et crèches vivantes médiévales, mais dont la vogue et la diversité d'usage atteignent leur apogée au XIX siècle. Le principe est de faire incarner des compositions picturales (mais la source peut varier: parfois, il s'agit de sculptures, de gravures, ou même de descriptions littéraires) à un groupe de modèles vivants tenant la pose devant un public. Ces imitations prenaient place dans les salons mondains et fêtes privées, à titre de cérémonies ludiques et didactiques où l'on aimait participer, admirer, reconnaître; mais aussi sur la scène des théâtres, où, dès la seconde moitié du XVIII ${ }^{\mathrm{e}}$, dans la ligne des préceptes de Diderot, nombre de pièces faisaient culminer leur action par l'immobilisation des acteurs dans la disposition exacte de célèbres tableaux peints. Et ce n'est pas là l'apanage unique des Mariage de Figaro ou des Brutus: le vaudeville s'en fait une spécialité, car le tableau vivant existe également comme divertissement populaire. On le croise dans les cirques sous forme de "poses plastiques", dans les fêtes foraines sous des chapiteaux à l'enseigne de "musées vivants", dans les foires paysannes en guise de célébration culturelle patriotique. Enfin, la dimension licencieuse du tableau vivant comme légitimation de l'exhibition du nu lui fait connaître un destin sulfureux dans les cabarets. De la fin du XVIII ${ }^{\mathrm{e}}$ au début du XX $\mathrm{X}^{\mathrm{e}}$ siècle, c'est donc une forme de spectacle omniprésente et incontournable 2 . Si la pratique semble ensuite disparaître et sombrer dans un oubli généralisé, certains artistes, notamment dans le champ du cinéma, continueront de cultiver le motif en le retravaillant et l'actualisant. C'est le cas, exemplaire, de Raoul Ruiz ${ }^{3}$.

\section{Toutes les dimensions du tableau vivant ruizien}

Le cinéma de Raoul Ruiz s'est, de fait, approprié le tableau vivant de dif- 
férentes manières, explorant à peu près toutes les ressources esthétiques du motif:

- Sa valeur emblématique de "simulacre» dans la lignée de Pierre Klossowski, dont les essais et romans convoquant le tableau vivant ont inspiré le premier film de Ruiz à user du procédé, L'Hypothèse du tableau volé (France, 1979). Raoul Ruiz est l'un des rares cinéastes à être fondamentalement anti-bazinien, revendiquant l'aspect construit et artificiel de l'image cinématographique. A cet égard, le tableau vivant, en tant qu'exercice d'imitation, représentation de représentation, jeu sur la citation et l'artifice, actualise parfaitement la conception du cinéaste pour qui "toute image n'est qu'image d'image" et s'engendre indéfiniment, "traduisible à tous les codes possibles", les "nouveaux codes" étant "générateurs d'images, elles-mêmes génératrices» $\mathbf{4}$. Un tel motif semble ainsi incontournable dans ses expérimentations filmiques marquées d'une fascination pour "le fait de prendre des lieux communs [artistiques] et de les retravailler»5.

- Son caractère de "pont entre les arts" pour reprendre les termes de Marie-Hélène Mello qui a étudié l'usage du tableau vivant dans L'Hypothèse du tableau volé6. Selon elle, le tableau vivant ruizien doit être avant tout envisagé en termes d'«image relationnelle» (ou "interimage») qui "exprime à la fois la rencontre, la contradiction, le décalage et l'entre-deux de plusieurs pratiques artistiques et des discours qui leur sont propres", questionnant les "propriétés de l'image et la spécificité du médium cinématographique» 7 . Ce paragone s'inscrit dans le cinéma de Ruiz qui se fonde sur une conception extrêmement libre de l'«adaptation" (d'histoires, de textes, de spectacles et d'images) - sans compter le fait que l'activité du cinéaste chilien s'étend également au théâtre, à la littérature, à l'installation artistique, etc.

- Enfin, sa temporalité paradoxale. Exercice d'immobilité, le tableau n'est vivant que s'il réinsuffle une certaine - mais infime - part de mouvement dans la pose. Ce paradoxe essentiel a clairement été décrit par Ruiz :

"Inévitablement, les modèles du tableau vivant bougent un peu, imperceptiblement. Ils doivent en permanence faire des efforts pour récupérer la 'pose`. Ils tournent sans cesse autour de cette pose qui les appelle mais se dérobe. » $\mathbf{8}$

Cette tension entre mouvement et immobilité ne prend que plus de résonance au cinéma, où l'«arrêt» du geste menace l'«arrêt» du film. Ainsi, et comme l'ont démontré d'autres films ${ }^{9}$, le tableau vivant est un lieu privilégié d'expérimentations sur la temporalité de l'image cinématographique. Or, pour le cinéaste chez lequel Christine Buci-Glucksmann a reconnu
4 Raoul Ruiz, Poétique du cinéma 1 (Miscellanées), Editions Dis voir, Paris, 1995, p. 53.

5 Michel Ciment, Hubert Niogret, Paulo Antonio Paranagua, "Entretien avec Raoul Ruiz", in Positif, n² 274, 1983, republié dans Raoul Ruiz. Entretiens, Hoëbeke, Paris, 1999, p. 42.

6 Marie-Hélène Mello, "Le tableau vivant d'après Raoul Ruiz. Une esthétique de l'interimage ^", dans Richard Bégin, Myriam Dussault et Emmanuelle Dyotte (éd.), La Circulation des images. Médiation des cultures, L'Harmattan, Paris, 2006, pp. 139-151.

7 /d., p. 140.

8 Raoul Ruiz, Poétique du cinéma 1, op. cit., p. 51.

9 Le court métrage de Pasolini La Ricotta (Italie, 1962) explore par exemple ces paradoxes dans toute leur dimension tragicomique, n'aboutissant à l'immobilité que par la mort du figurant et l'arrêt sur image. 
10 Cyril Béghin, "Une métaphysique de l'indécidable. Entretien avec Christine Buci-Glucksmann", dans Théâtres au cinéma. Raoul Ruiz, Magic Cinéma, Bobigny, 2003, p. 58.

11 Raoul Ruiz, Poétique du cinéma 1, op. cit., p. 51.

12 Youssef Ishaghpour, "Entre miroirs et tableaux. Raoul Ruiz: L'Hypothèse du tableau volé", dans Cinéma contemporain, de ce côté du miroir, La Différence, Paris, 1986, p. 206. une «esthétique du suspens» $\mathbf{1 0}$, ce paradoxe de la pose entraîne avec lui un enrayage temporel plus vertigineux encore:

«Nous savons que les peintres utilisaient aussi des modèles vivants. [Cette] tension physique [qu'éprouvent les modèles du tableau vivant dans la pose], [c'est] la même qu'ont dû éprouver les modèles originaux. Une telle intensité est comme un pont qui relie les deux groupes de modèles. Les petits mouvements des modèles d'origine, figés dans la peinture, sont alors reproduits par les modèles dans le tableau vivant. Les premiers modèles sont, en un sens, réincarnés dans le tableau vivant, ou du moins est-ce la tension qui se trouve réincarnée. Dans de tels gestes réincarnés, certains philosophes comme Nietzsche et Klossowski ont vu une illustration, peut-être même une preuve, de l'éternel retour. »11

Ces «réincarnations" pourront prendre un caractère proprement fantastique ou funèbre chez ce cinéaste qui conjugue le vivant avec la mort et affectionne les figures de fantômes. En tout cas, le tableau vivant y incarne un "trou noir» temporel qui, inscrit dans "l'après", ressuscite "l'avant» du tableau, actualisant une conception de la temporalité non plus linéaire mais cyclique, chère à Raoul Ruiz pour qui la linéarité, quelle qu'elle soit (narrative, causale, spatiale), est un paradigme à combattre.

Si ces facettes - toutes inextricablement liées - sont expérimentées par le cinéaste, c'est encore une autre dimension du tableau vivant ruizien que je vais étudier ici, à savoir sa nature scénographique de dispositif du regard. Dans le cinéma de Raoul Ruiz, le tableau vivant, acte de mise en scène dans la mise en scène, m'apparaît être un moyen privilégié de thématiser la perception spectatorielle et de l'expérimenter dans des voies inédites. Le canevas perceptif traditionnel s'y déconstruit au profit d'une vision renouvelée, modifiée, voire libérée.

\section{Un terrain d'expérimentation du point de vue}

Le tableau vivant se définit avant tout comme un dépassement de la planéité de l'image peinte en faveur de son déploiement tridimensionnel, avec l'accès à la liberté de regard que cela implique:

"Le moyen technique du tableau vivant [...] est en somme une sorte de cinéma en suspension, avec la possibilité de circuler dans le champ, de changer d'angle et de voir ce qui à première vue n'est pas visible. »12

Cette définition de Youssef Ishaghpour qui associe les spécificités du tableau vivant à celles du cinéma s'applique parfaitement à l'usage du motif chez Raoul Ruiz. Dès ses premières captations de danse, le cinéaste explique vouloir "montrer ce que le spectateur de danse ne voit 
pas, les danseurs vus d'en haut, ou d'en bas, ou leurs visages, qu'on ne voit jamais lorsqu'on est dans une salle», établissant que «le changement d'axe n'est pas décoratif, [mais] crée une implantation nouvelle»13. En mettant en espace le tableau vivant et en l'arpentant au prisme de «l'œil baroque de sa caméra»14, Ruiz pourra (comme avec la danse) révéler la face cachée du tableau peint, libérer le spectateur du point de vue pictural unique et arrêté. En effet, passionné et fin connaisseur des innombrables théories qui forment l'histoire de la perception, Raoul Ruiz estime que l'énigme fondamentale du cinéma réside dans la question: "Où suis-je, où est ma place, où est le point de vue?»15. Il revendique le fait de placer sa caméra "jamais là où elle devrait être", afin de brouiller la vision conventionnelle de l'espace au profit d'une "perception éparpillée» en accord avec la sélectivité du cadre et la discontinuité du médium ${ }^{\mathbf{1 6}}$. En vue de montrer que le tableau vivant est le lieu idéal de cette expérimentation perceptive, mon étude parcourra trois films du cinéaste qui, à quelque dix ans d'intervalle (ce qui démontre la permanence du motif), usent de ce dispositif de vision pour mettre en abyme la perception filmique et appeler une certaine forme de "spectature": contemplative dans L'Hypothèse du tableau volé, pulsionnelle dans Généalogies d'un crime (France, 1997), enfin onirique dans Klimt (Autriche/France/Allemagne/Angleterre, 2006). Tous ces paradigmes perceptifs rompent avec le modèle cognitif linéaire attendu d'un spectateur de film classique.

\section{L'Hypothèse du tableau volé ou l'initiation à la contemplation}

La première fois que le motif apparaît dans la filmographie de Ruiz, c'est de manière résolument explicite, dans un film qui devait précisément s'appeler Tableaux vivants avant d'être renommé L'Hypothèse du tableau volé17. Ce film consiste en une promenade de la caméra, guidée par un collectionneur érudit et une voix off, à travers les reconstitutions tridimensionnelles, posées par des modèles vivants, d'une énigmatique et incomplète série de tableaux peints.

Le vertige temporel engagé par ces tableaux vivants est d'emblée annoncé: premièrement, un mystère historique brouille le rapport entre tableaux peints et tableaux vivants, dont on ne sait plus lesquels sont à l'origine des autres; deuxièmement, la perpétuelle mise en scène du collectionneur est une tentative de remonter, conformément à la dynamique d'éternel retour décrite par Ruiz, à la pose originelle des tableaux; troisièmement, il cherche une lecture linéaire et successive des toiles mais découvrira que "les mêmes gestes" sont répétés "de tableaux en tableaux»18. Et si la temporalité tourne court, l'espace, lui, se
13 Hervé Le Roux et Guy Scarpetta, "Raoul Ruiz, vers un cinéma du dix-huitième siècle [interview]", in Art Press, n 112, 1987, p. 40.

14 Christine Buci-Glucksmann définit ainsi la manière de filmer de Ruiz, "qui nous place toujours dans la perplexité redondante et mensongère d'un voir multiple, double, anamorphique et miroitant, à facettes." Cf. "L'œil baroque de la caméra", dans Christine Buci-Glucksmann, Fabrice Revault d'Allonnes, Raoul Ruiz, Editions Dis Voir, Paris, 1987, p. 10.

15 Philippe Carcassonne, "Entretien avec Raoul Ruiz", in Cinématographe n 48, 1979, p. 48.

16 Raoul Ruiz, "Entretien avec Pierre-Alexandre Nicaise et Eric Lebot, 9 mars 2007 ", sur www. lecinemaderuiz.com, p. 1.

17 II est annoncé en ces termes par Pascal Bonitzer en mars 1978 dans les Cahiers du cinéma, $n^{\circ} 286,1978$, p. 73 . Ruiz lui-même s'explique sur ce changement de titre dans $D u$ Chili à Klossowski. Entretien avec Raoul Ruiz par Jerôme Prieur (documentaire de 30 min. en bonus de l'édition DVD du film).

$1855^{\mathrm{e}}$ minute, réplique de Jean Rougeul. 
19 La voix off le formule explicitement à la $44^{e}$ minute du film, soulignant que "le collectionneur nous demande, une fois de plus, d'oublier le fil conducteur pour nous concentrer dans la mise en scène du tableau ".

20 "Raoul Ruiz, I'homme qui regarde", in Art Press, n 172,1992 , p. 14.

21 Ce tableau vivant est repris au récit homonyme de Klossowski, où cette scène mythologique est envisagée comme un dispositif complexe de vision. Voir Pierre Klossowski, Le Bain de Diane, Gallimard, Paris, 1980.

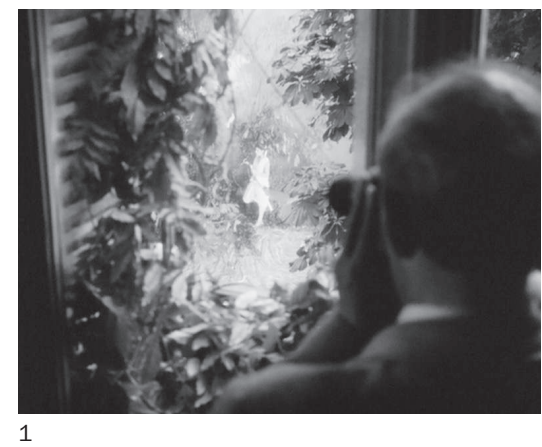

déploie. L'exploration proposée par le film est de fait bien plus topologique que chronologique19. Ruiz explique ne s’être intéressé au motif pictural du tableau que lorsqu'il a pu y reconnaître un dispositif de mise en scène, mettant en interaction des corps et des gestes:

"J'avais toujours refusé l'idée qu'un tableau puisse être à l'origine d'un film. [...] Car je voulais filmer le mouvement et l'espace tandis qu'un tableau travaille sur des matières. C'était mon idée et [...] Klossowski m'a appris que les tableaux ne sont pas seulement des représentations de choses mais que ce sont aussi des cérémonies et donc des sources de mises en scène. » $\mathbf{0}$

C'est donc bien l'espace que parvient à filmer Raoul Ruiz dans ces tableaux devenus pure mise en scène. Le premier que l'on découvre, $L e$ Bain de Diane ${ }^{\mathbf{2 1}}$, annonce d'emblée la manière dont la caméra va briser le cadre pictural et l'immobilité du point de vue. Tandis que le collectionneur s'immobilise derrière une fenêtre (fig. 1) et enserre son regard dans des jumelles qui lui donnent presque exactement l'angle de vue imposé par le tableau peint (fig. 2-3), la caméra va au contraire «s'échapper» de ce dispositif albertien, "traverser» le cadre plane de la fenêtre pour s'insinuer au cœur du tableau vivant, et découvrir ce même «instant»
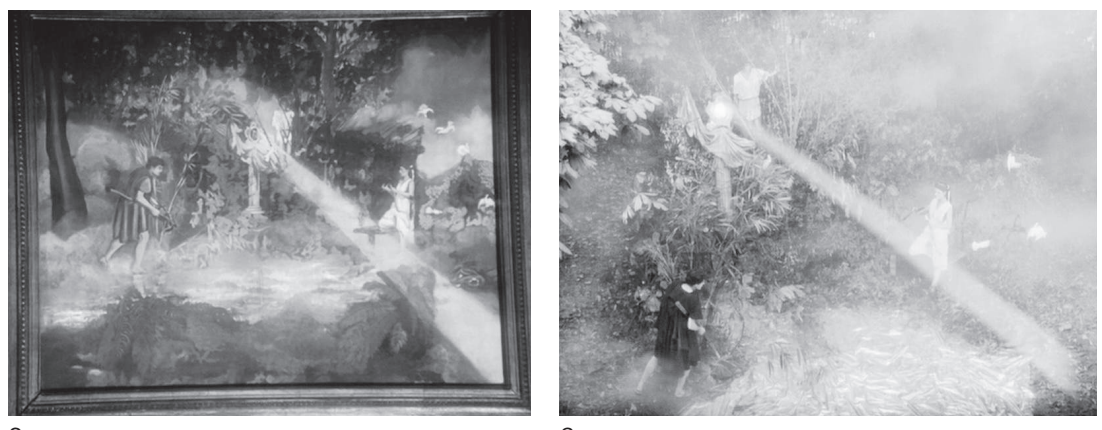

3 


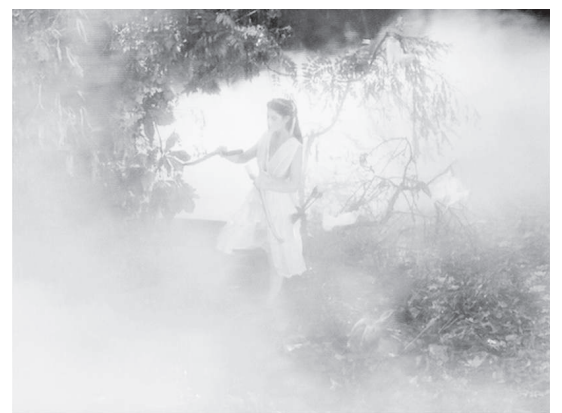

4

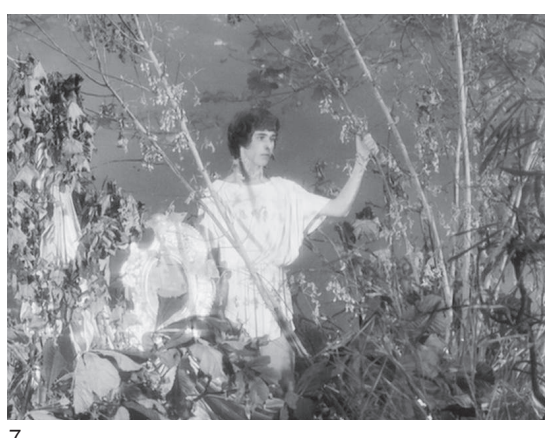

7

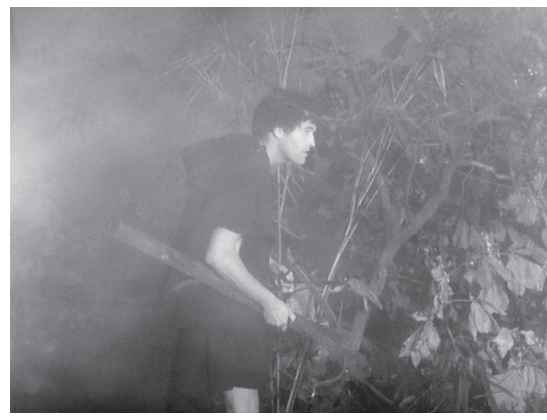

10

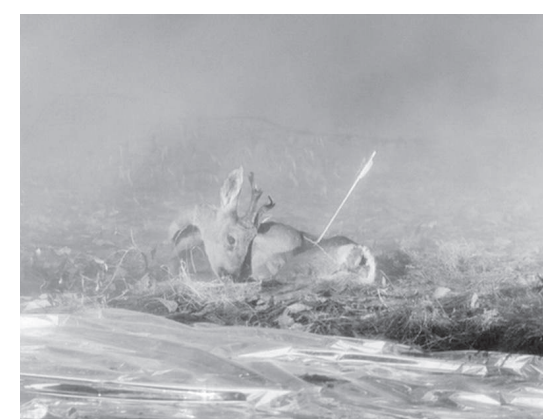

5

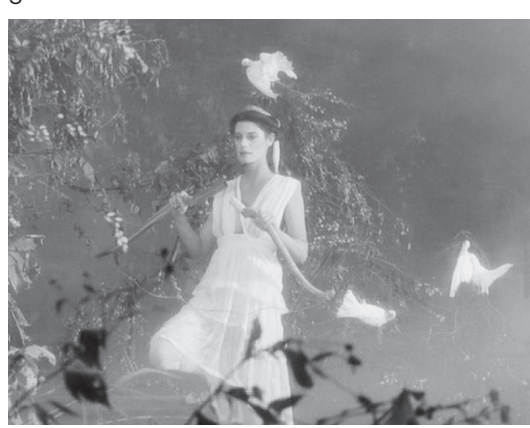

8

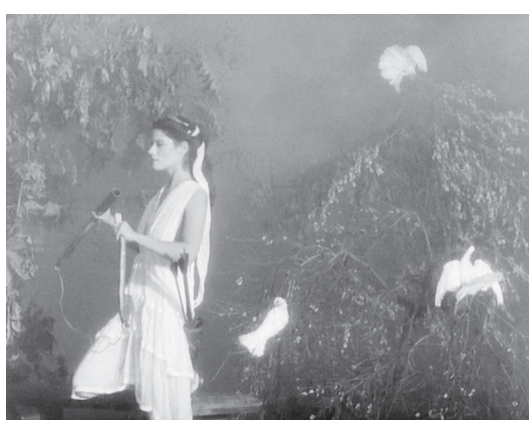

11
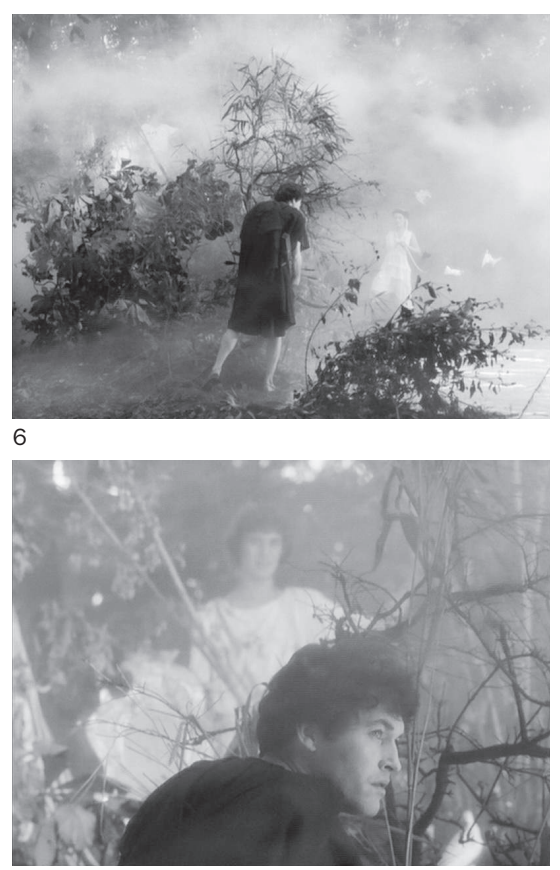

9

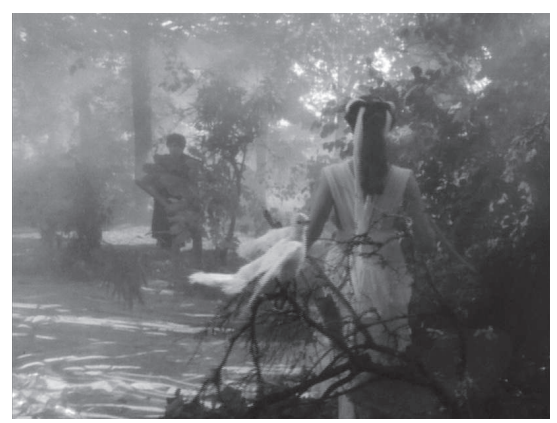

12

sous plus de dix points de vue aux échelles de plans toujours différentes (fig. 4-12), sans compter les mouvements de caméra, les changements de focale et d'éclairage (via notamment les évolutions de la brume artificielle). Dans les tableaux vivants suivants, le collectionneur lui-même entrera dans les tableaux et déambulera entre les modèles, tout à la fois guidant et égarant notre regard par ses déplacements (fig. 13-14). Il nous permettra ainsi de voir les tableaux vivants non seulement sous d'autres facettes mais aussi sous d'autres éclairages; il pourra même intimer le ralenti d'un geste, animant ainsi les poses. L'exploration, par la caméra, de tous ces points de vue dont "simultanés", chacun saisissant le même 


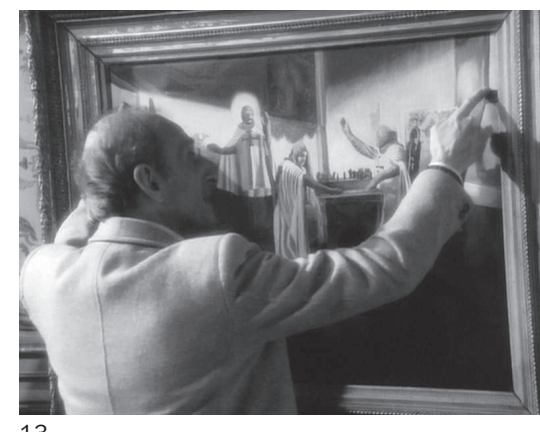

13

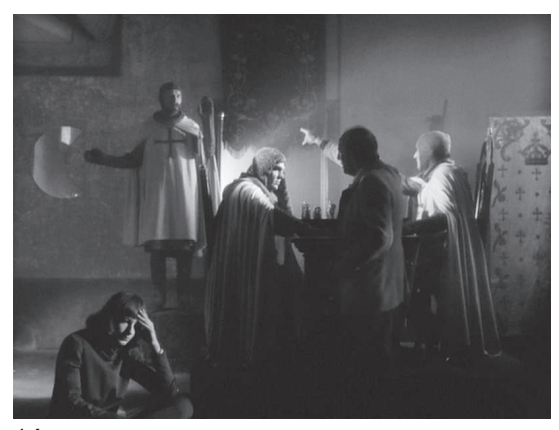

14
22 "[J'ai choisi Rougeul parce que] j'avais senti qu'il aimait bien parler, avec des phrases longues. C'est rare de voir un Français qui s'étende comme ça avec des phrases intercalées, en reprenant les mots et les phrases [...], changeant le sens pendant qu'il parle, [...] en faisant une espèce de discours cubiste." (Du Chili à Klossowski. Entretien avec Raoul Ruiz par Jerôme Prieur, documentaire cité, $20^{\mathrm{e}}$ minute). Notons qu'au fondement du cubisme réside toute une réflexion sur la perception (visuelle, mémorielle, émotionnelle) et sur la manière dont elle se construit. Voir à ce sujet la définition de René Berger pour qui les cubistes donnent à voir la "gestation discontinue de notre perception", dans Connaissance de la peinture, tome VII, "Espace, perspective, vision ", Centre international des arts/Club français du Livre/Novorop, Paris/Monte-Carlo/Lausanne, 1963, p. 354.

23 C'est Nicolas Poussin qui relance et popularise cet usage, en ayant "comme habitude de disposer dans une boîte à perspective des petits modèles en cire ou en terre, recouverts de draperie, de ses personnages, et de les déplacer jusqu'à ce que la disposition lui convienne" (Louis-Antoine Prat, "CEuvres choisies: L'Extrême-Onction. Nicolas Poussin, 1644 - Département des Arts graphiques", www.louvre.fr). L'Académie, qui érige la peinture de Nicolas Poussin en parangon, prônera cette technique dite "plastique", dont René Passeron a montré combien elle préfigurait les tableaux vivants: "dans cet espace scénique (cube avec édicules chez Giotto, ou plateau devant toile de fond chez Piero della Francesca), on prend des poses. Un peu de maniérisme et ce seront des 'poses plastiques?." (René Passeron, L'CEuvre picturale et les fonctions de l'apparence, Vrin, Paris, 1986, pp. 34-35). instant éternisé du tableau vivant, n'est pas sans évoquer une approche cubiste - c'est d'ailleurs précisément en termes de "discours cubiste» que Ruiz décrira le dialogue du film 22.

En outre, ce déploiement des points de vue sur les figures du tableau permet de revivre les recherches optiques qui ont précédé l'établissement du cadre pictural. En effet, dans la peinture académique (qui est concernée ici), en amont même des séances de pose, le peintre cherchait sa composition et son regard via de petites figurines en bois ou en cire, dont il testait les poses dans une boîte dite «à perspective» qui lui permettait d'examiner sa composition sous tous ses aspects, avant de délimiter un angle de vue ${ }^{23}$. Cette modalité de mise en scène, et surtout de "mise en œil», est d'ailleurs explicitement thématisée dans L'Hypothèse du tableau volé, où le collectionneur, avant d'explorer les «perspectives» du tableau grandeur nature, joue à manipuler des petites figurines (fig. 15). Cette exploration de la "boîte à perspective" (que Francastel a significativement renommée "cube scénographique»), répond donc très exactement à la démarche cinématographique de Ruiz, pour qui "construire un décor», c'est s'accorder "la possibilité de placer la caméra dans des milliers d'endroits", en se situant "à la périphérie d'[une] sphère visuelle»

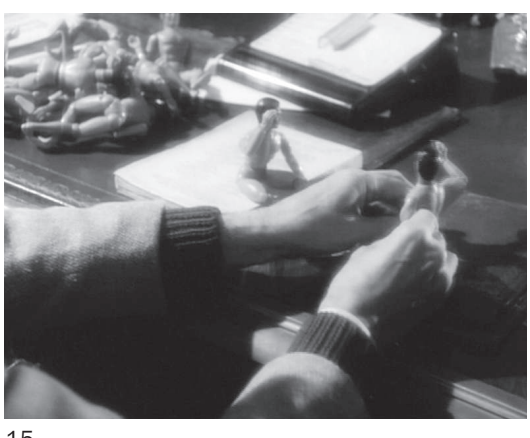


qui nous fait rejoindre «la variété de la vision de Dieu»24. Difficile de mieux décrire le fonctionnement scénographique et démiurgique de la boîte à perspective, et du tableau vivant dans L'Hypothèse du tableau volé.

Cette démultiplication des angles de vue entraîne le spectateur à concevoir la richesse des variations de sa perception. Plusieurs commentateurs, à l'instar de Philippe Carcassonne, ont reconnu l'impact de ce film sur la "faculté de regard"du spectateur, qui prend conscience de sa capacité à «s'approprier les formes» et à «transpercer les images» $\mathbf{2 5}$. Le film de Ruiz vise en effet à éveiller le sens visuel du spectateur et à aiguiser sa manière de décrypter les plans, pour le faire entrer dans une appréciation contemplative et personnelle de l'image cinématographique, par opposition à la perception addictive, immédiate et superficielle prônée par le cinéma hollywoodien $\mathbf{2 6}$. Et si L'Hypothèse du tableau volé est sûrement son film le plus explicite quant à cette "formation contemplative», cela est peut-être directement lié au motif des tableaux vivants. En effet, dans les salons du XIX ${ }^{\mathrm{e}}$, le tableau vivant faisait figure d'"exercice de contemplation». En tant que jeu mondain, il était un défi lancé au regard et à la culture visuelle du public, qui devait reconnaître l'original, comparer mentalement les versions peinte et vivante, enfin "dire son avis sur la manière dont le tableau [était] exécuté» $\mathbf{2 7}$. Il s'agissait donc d'exercer son «œil», et d'en parler ${ }^{28}$. L'usage du tableau vivant dans L'Hypothèse du tableau volé réactualise ainsi pleinement ses vertus historiques d'initiation à la contemplation.

Et si ce film transforme l'exercice de mise en scène en expérience de regard et le statisme des poses en mobilité du point de vue, cela était déjà le cas au XIX ${ }^{\mathrm{e}}$ siècle puisque - et c'est là notre dernier argument le tableau vivant était souvent exposé au public de manière à mettre en mouvement son regard, suivant un dispositif précisément décrit par Victor Hugo:

"Le rideau s'entrouvrait, et ils exécutaient un tableau. Pour cela, ils étaient montés et disposés dans des attitudes immobiles sur un large disque en planches, lequel tournait sur un pivot. Un enfant de quatorze ans couché dessous, sur un matelas, suffisait à manœuvrer ce disque. [...] Quand le disque avait achevé un tour et montré les statues sous toutes leurs faces au public entassé dans la salle obscure, le rideau se refermait, on disposait un autre tableau, et la chose recommençait le moment d'après. » $\mathbf{2 9}$

Les spectateurs «entassés dans la salle obscure» examine l'image en mouvement et «sous toutes ses faces» : le tableau vivant semble définitivement préfigurer et emblématiser la perception cinématographique telle que la travaille Raoul Ruiz ${ }^{30}$.
24 Pascal Bonitzer et Serge Toubiana, "Entretien avec Raoul Ruiz", op. cit., pp. 21-22.

25 Philippe Carcassonne, "L'Hypothèse du tableau volé, Raoul Ruiz", in Cinématographe, n 48, 1979, p. 49.

26 Ruiz explique dans son "Entretien avec Pierre-Alexandre Nicaise et Eric Lebot, 9 mars 2007 " (op. cit., p. 1) vouloir sauver "l'aspect contemplatif du cinéma" qui serait en train de disparaître, étouffé par la "fausse idée" de la perception filmique propre à Hollywood où tout mouvement des yeux du spectateur face à l'écran est condamné comme une "distraction" néfaste.

27 F. M. Von Grimm cité dans Denis Diderot, Salons, tome II, "Salon de 1765 ", Clarendon Press, Oxford, 1960, p. 155, cité par Vouilloux, op. cit., p. 21.

28 Le tableau vivant connaissait même un usage purement didactique, en étant cultivé comme exercice pédagogique dans le cadre de l'"éducation visuelle" des enfants (Kirsten Gram Holmström, op. cit., p. 217).

29 Victor Hugo, Choses vues. Souvenirs, journaux, cahiers, tome I, "1830-1848", Gallimard, Paris, 1997, p. 391, cité par Vouilloux, op. cit., p. 30.

30 II semble toutefois que ce dispositif du plateau tournant n'était utilisé que pour les reconstitutions de sculptures, les imitations de peintures étant expressément disposées de sorte à restituer le point de vue pictural immobile, ainsi qu'en témoigne l'anecdote (fictive ou non) de Goethe racontant que face à un tableau vivant dont le principal et magnifique modèle posait de dos, imitant une composition peinte par Terborch (La Remontrance paternelle, 1655), le public "joyeux drille" se mit à crier "tournez s'il vous plaît!", mais sans succès. (Johann Wolfgang von Goethe, Les Affinités électives, Flammarion, Paris, 1992 [1809], p. 220.) Rappelons cependant que dans les expositions plus populaires du tableau vivant, l'accès à d'autres points de vue est bien l'un des enjeux majeurs de l'imitation, notamment dans les explorations érotiques du motif, où le caché vise expressément à être dévoilé, ou dans les "musées vivants" forains, où les spectateurs devaient déambuler entre les modèles. 


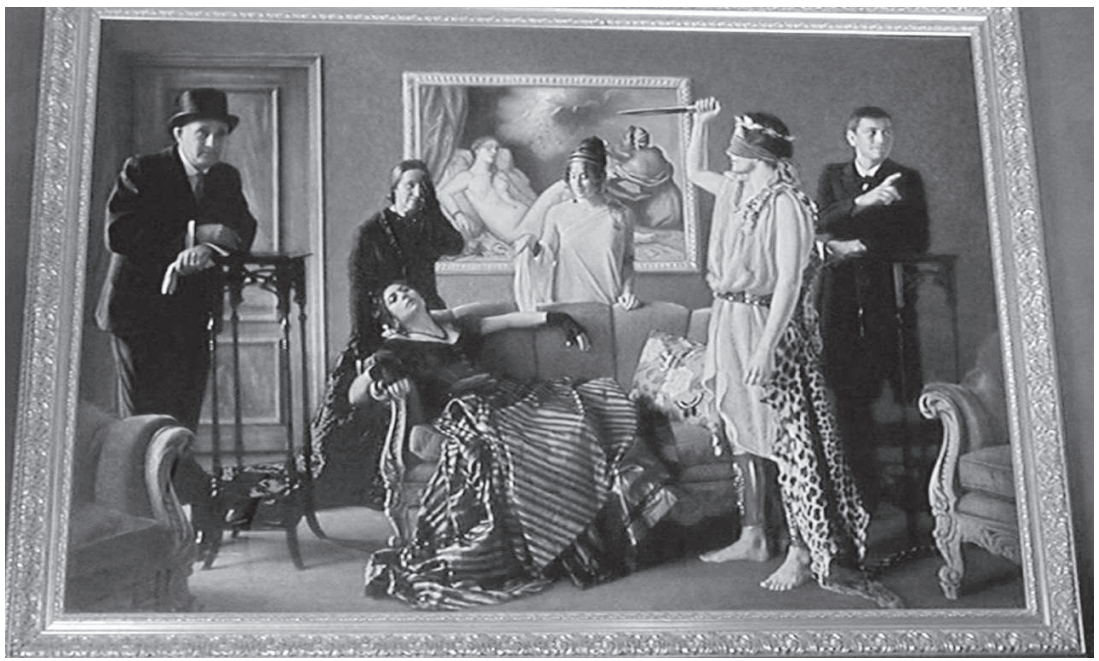

31 Notons que c'est là l'exacte définition de "l'instant prégnant", notion établie par Lessing au XVIII ${ }^{e}$ siècle sur le modèle de l'art grec afin de déterminer l'attitude dans laquelle figer la pose, en s'intéressant à la psychologie du spectateur et en tirant profit des paradoxes temporels de ce moment qui en même temps doit être nul et durer à jamais: "Timomaque n'a pas peint Médée à l'instant même où elle tue ses enfants, mais quelques instants avant, lorsque l'amour maternel lutte encore avec la jalousie. Nous prévoyons la fin de cette lutte; nous tremblons d'avance de voir bientôt Médée livrée toute à sa fureur, et notre imagination devance de bien loin tout ce que le peintre pourrait nous montrer dans ce terrible instant. [...] Pour ses compositions, qui supposent la simultanéité, la peinture ne peut exploiter qu'un seul instant de l'action et doit par conséquent choisir le plus fécond, celui qui fera le mieux comprendre l'instant qui précède et celui qui suit." (Gotthold Ephraim Lessing, Laocoon, Paris, Hermann, 1990 [1766], pp. 56-57).

\section{Généalogies d'un crime ou l'analyse du regard}

L'Hypothèse du tableau volé n'est pas le seul film de Ruiz à réactualiser les vertus scénographiques et contemplatives du tableau vivant; le motif réapparaît explicitement dans Généalogies d'un crime, utilisé par les protagonistes comme un jeu de rôle psychanalytique. Le court-circuit temporel qu'il engage est central : l'enjeu même de l'exercice est de confronter un patient à sa prédestination criminelle. Ainsi le tableau est-il une allégorie de l'acte criminel futur du patient, ou plus précisément du moment situé «juste avant le geste irréparable» - l'instant ultime où il peut encore choisir de déclencher ou retenir l'action suspendue, mise en "pose»31. L'exercice du tableau vivant permet ainsi de faire "répéter» au patient le passé immédiat de son futur: le temps s'enraie donc plus que jamais dans cette pose déclinée au "futur antérieur». Et, une fois encore, le rapport au tableau peint (que nous voyons subitement apparaître, plein cadre, à l'écran, sans aucune explication [fig. 16]) est entouré de mystère: nous ne pouvons savoir lequel est le modèle de l'autre. On se rendra d'ailleurs compte qu'ils entretiennent plutôt un rapport spatial d'«inclusion", le tableau peint étant accroché dans la pièce même où l'on joue le tableau vivant (on voit notamment son reflet dans un miroir qui domine, en son centre, la composition vivante [fig. 17]), générant une mise en abyme vertigineuse où les simulacres s'engendrent et s'incluent.

Mais au-delà d'une expérience temporelle déconstruisant toute linéarité et notion de destinée, ce tableau vivant expérimente la contemplation, mettant en place un véritable rituel du regard. Le patient doit d'abord demeurer derrière une vitre sans tain, d'où il peut regarder le 


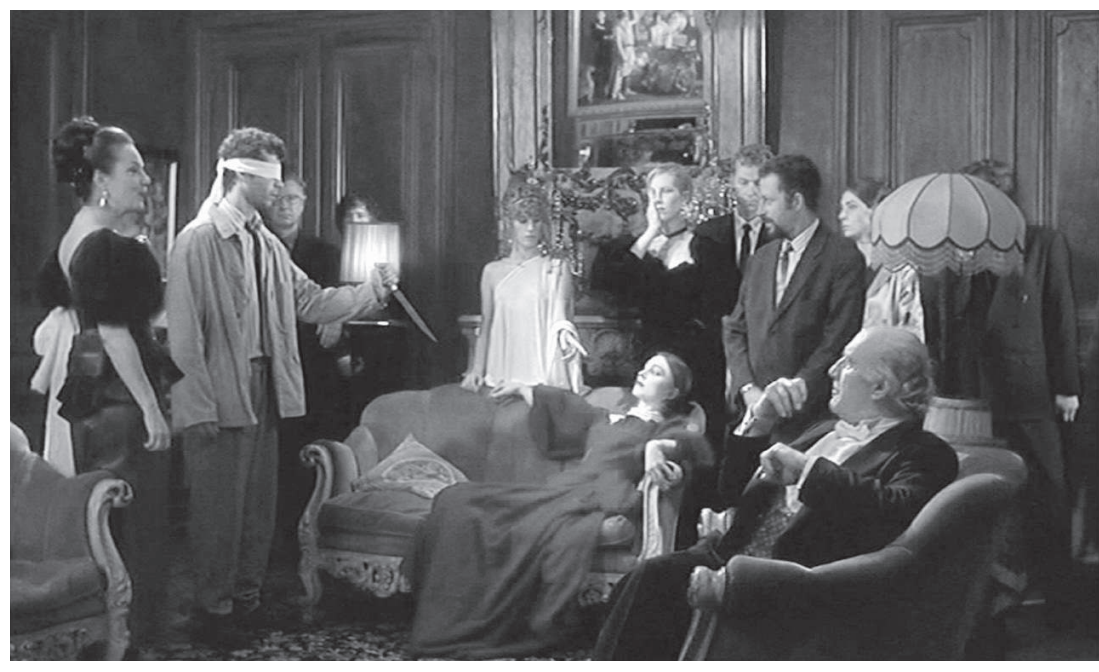

tableau vivant mis en pose, son rôle y étant tenu par un "double théâtral». Tous les modèles, en plus d'être immobilisés, sont aveuglés par un bandeau sur les yeux, l'acte de voir étant réservé au seul patient. Puis il doit quitter ce point de vue extérieur et omniscient, "traverser le miroir» et entrer dans le tableau, en y prenant la place de son «double». Dès lors les rapports de regards s'inversent: tandis que lui revêt son bandeau, n'ayant plus le droit de voir, les autres l'enlèvent, et le regardent. Le tableau vivant fonctionne donc comme un dispositif visuel, le patient étant invité à contempler cet instant, à en être le "voyeur", à s'y projeter, à en saisir toutes les facettes, puis à s'y aveugler. Le but est d'exorciser la "pulsion criminelle" pour la transmuer en "pulsion scopique». Et ce n'est bien sûr pas un hasard si le tableau vivant, qui permettrait l'assouvissement de cette pulsion, est mis en scène ici de sorte à refléter toutes les composantes du cinéma : spectateur, écran, projection (dans un temps et un personnage "doubles»), metteur en scène, figurants, jeux de rôle, spectacle cyclique.

La mise en abyme incite à étendre la dimension psychanalytique de l'exercice à l'ensemble des principes cinématographiques. De fait, nombre de théories ont exploré les aspects freudiens, lacaniens, ou jungiens de cet appareil de projection-simulation-voyeurisme, et interprété l'acte perceptif du spectateur en termes pulsionnels 32 . Utiliser le motif du tableau vivant pour refléter cette dimension psychanalytique, voyeuriste et licencieuse du regard spectatoriel est plus que légitime, puisque cela correspond à la réalité historique de cette pratique où la convoitise érotique pour ces corps "exposés" s'est vite mêlée à la contemplation
32 Pour un aperçu historiographique de la question, voir notamment Mireille Berton, "Freud et l'intuition cinégraphique: psychanalyse, cinéma et épistémologie", in Cinémas, vol. 14, $n^{\circ}$ 2-3, 2004, pp. 53-73. 
33 Réplique de Camila Mora, $80^{\mathrm{e}}$ minute.

$34 \mathrm{~A}$ la $74^{\mathrm{e}}$ minute, le psychanalyste (Michel Piccoli) s'écrie: "Cette technique ne doit rien à Moreno, absolument rien!" Sur le "théâtre spontané" de Moreno, et les méthodes de "théâtrothérapie ", voir notamment Serge Minet, Du divan à la scène. "Dans quelle pièce je joue?", Mardaga, Liège, 2006.

35 Voir notamment Laurent Assoun, "L'inconscient du crime. La "criminologie freudienne " " in Recherches en psychanalyse, n 2004/2 ("Le crime"), pp. 23-39.

36 "Du Chili à Klossowski. Entretien avec Raoul Ruiz par Jerôme Prieur", documentaire cité, $26^{\mathrm{e}}$ minute. artistique. Le film thématise explicitement cette dynamique voyeuriste: l'un des modèles révélera que l'exercice "dérapait» régulièrement en «partouze»33. De plus, la relation qu'entretient le patient-spectateur avec sa victime-modèle est largement teintée de désir incestueux. S’il repose sur une forme de voyeurisme, le tableau vivant comme thérapie psychanalytique n'a cependant pas de bases historiques réelles. On peut toutefois songer à des pratiques similaires, qui ont exploré les vertus psychanalytiques de la mise en scène, du jeu de rôle et du dédoublement de point de vue, à l'instar du psychodrame inventé par Jacob Levy Moreno, que le film cite comme une référence ${ }^{34}$.

Au-delà de sa dimension psychanalytique, ce tableau vivant invoque une autre tradition, d'ordre policier. En effet, Généalogies d'un crime montre l'enquête menée par une avocate (Catherine Deneuve) sur le meurtre commis par son client (Melvil Poupaud). Et c'est le psychanalyste qui a inventé ce «traitement au tableau vivant» (Michel Piccoli) qui, en reconstituant la cérémonie, lui révèle comment l'acte criminel du patient y a surgi. Ainsi le tableau vivant fonctionne-t-il à tous les niveaux (dans son sujet allégorique, dans son usage thérapeutique et dans sa mise en scène filmique) comme la reconstitution d'une scène de crime. Or la criminologie - qui n'est pas sans liens avec la psychanalyse $\mathbf{3 5}$ - passe bel et bien par de telles mises en scène, dites "reconstitutions des faits". Certes le tableau vivant au sens strict n'est pas exploité, mais il s'agit aussi de reconstruire une scène exactement comme elle s'est produite, de sorte à mieux la voir, souvent en y arrêtant le temps pour explorer l'espace dans tous ses détails, en usant de mannequins ou de figurants aptes à tenir la pose. Enfin et surtout, la reconstitution se donne comme un spectacle, devant un public de témoins auprès de qui on espère provoquer des flashs mémoriels, et devant des observateurs aguerris aptes à percevoir tous les détails suspects. C'est ce même regard avide de mystères, d'indices, et de "mieux voir» que veut susciter le tableau vivant ruizien. C'était déjà celui convoqué dans L'Hypothèse du tableau volé, où, comme, Ruiz l'explique dans un entretien, «les tableaux sont composés d'après des illustrations de faits divers, publiées dans les revues populaires de l'époque» $\mathbf{3 6}$, autrement dit une imagerie de reconstitution criminelle. Cette avidité scopique prend une dimension plus complexe en activant une dimension refoulée dans le dispositif de Généalogies d’un crime, qui pourrait être renommé Généalogies de la "perception» d’un crime.

\section{Klimt ou les vertiges de la perception}

Le film que Ruiz réalise en 2006 sur la vie et l'œuvre du peintre Gustave Klimt pourrait faire accroire qu'il use du tableau vivant à la manière 
"clandestine" des biopics (les films biographiques sur des artistes), où l'on reconstitue par ce biais les scènes de pose soi-disant originelles des toiles. Mais, invalidant d'emblée toute comparaison de Klimt avec un biopic standard, le cinéaste qui connaît l'histoire et les enjeux du tableau vivant y travaille le motif de manière plus complexe et inattendue.

Il n'y a qu'un tableau vivant au sens strict dans Klimt, et il est extrêmement subreptice. Il intervient à la $77^{\mathrm{e}}$ minute du film, lorsque Klimt (John Malkovich) découvre qu'il a été pris au piège d'un "maîtrevoyeur", qui a orchestré des rencontres avec un mystérieux modèle en déterminant et en espionnant toutes les évolutions érotiques et picturales de ses rendez-vous à travers des miroirs sans tain. Klimt fait alors coulisser l'un de ces faux miroirs, derrière lequel il trouve la loge kaléidoscopique du fameux orchestrateur-voyeur encombrée d'autres miroirs, de paravents et de vitres sans tain s'ouvrant sur plusieurs chambres attenantes où évoluent des femmes nues et des couples dans une valse de faux-semblants où le regard du spectateur, comme celui de Klimt, se perd. A l'opposé, le maître des lieux, assis dans un fauteuil pivotant, accoudé à une batterie de longues-vues dont il use à volonté pour mieux observer toutes les facettes de "ses» spectacles, renvoie à un spectateur de cinéma omniscient, qui a fait du «tout voir» un «tout pouvoir». Or derrière l'une de ces vitres sans tain, selon un dispositif un peu similaire à Généalogies d'un crime, deux modèles tiennent pour lui la pose du tableau le plus connu de Klimt: Le Baiser (fig. 18-19). Immobiles jusqu'à ce que retentisse un carillon, ils se raniment alors et viennent saluer leur "public» (fig. 20-21). Nous assistons donc bien à un tableau vivant, où la pose est donnée à voir comme un spectacle autonome - d'ailleurs,
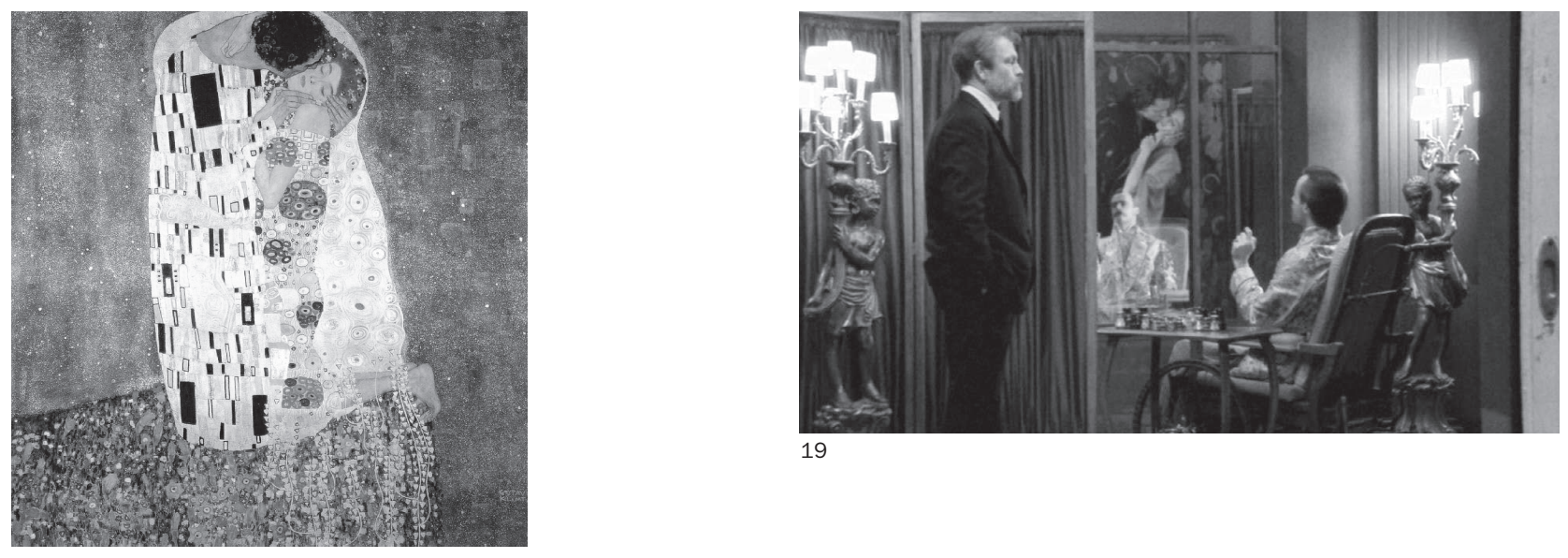


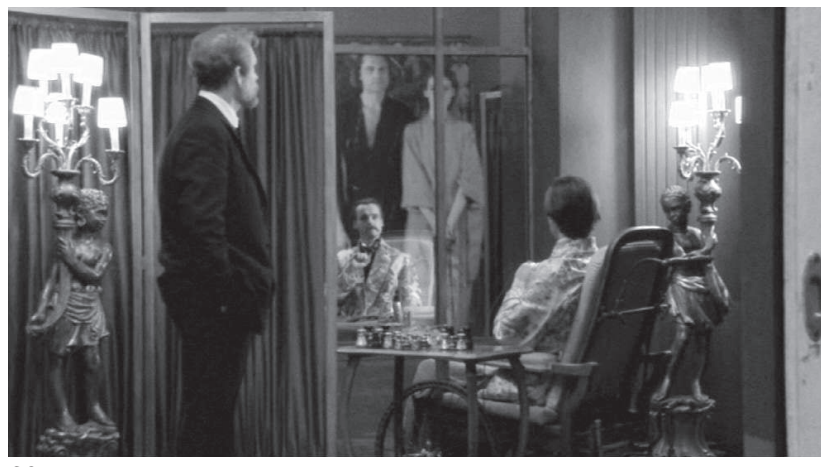

20

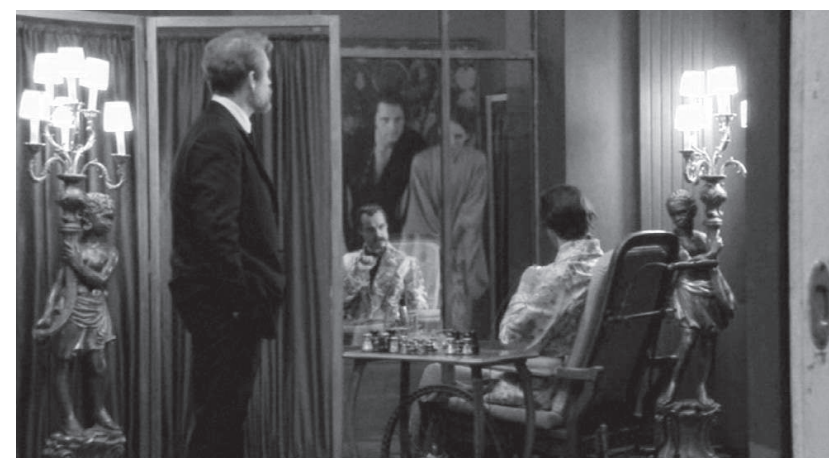

21

derrière un autre miroir sans tain, la même pose sera incarnée par d'autres modèles, enflammée cette fois par le mouvement passionné des amants (fig. 22). Il ne s'agit pas d'une reconstitution de la pose préparatoire du tableau: Klimt n'est ni en train de peindre ni même vraiment en train de regarder, pris dans la discussion avec son voyeur et dans le vertige des trompe-l'œil de la pièce. D'ailleurs le spectateur lui-même a du mal à distinguer le tableau vivant. Contrairement aux films précédents de Ruiz, la caméra ne vient pas le détailler sous différents points de vue. Au contraire, il est presque dissimulé dans l'image, relégué dans la profondeur indistincte de l'arrière-plan et «fondu» dans les reflets de la vitre sans tain.

D’autres «ébauches» de tableau vivant surviennent, de manière tout aussi furtive, comme à la $47^{\mathrm{e}}$ minute où, dans son atelier, Klimt est montré en train de peindre le tableau intitulé Les Amies et se voit interrompu par une visite, motivant un mouvement de caméra qui nous laissera entrapercevoir à l'arrière-plan les deux modèles du tableau qui tenaient la pose mais qui s'en défont aussitôt (fig. 23-24) 37. C'est encore le cas

37 Notons que Positif a produit une photographie de plateau de ce tableau vivant en le montrant plein cadre dans une pose parfaitement tenue, rendue ainsi à l'espace non fuyant et au point de vue fixe, unique et choisi de la photographie (voir Positif, n5 542, 2006, p. 3). dans la célèbre séquence qui met en scène une discussion entre Klimt et Midi dans l'atelier du peintre, au moment où il est occupé à appliquer des feuilles d'or sur une toile. L'espace y est travaillé de manière extrê-
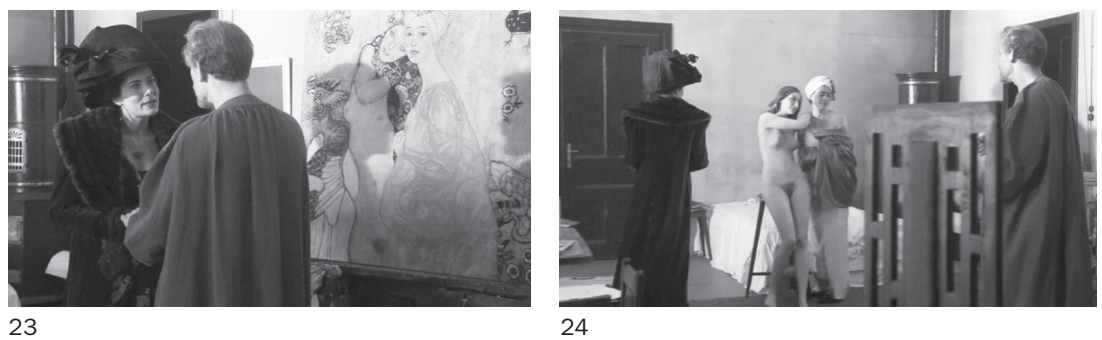


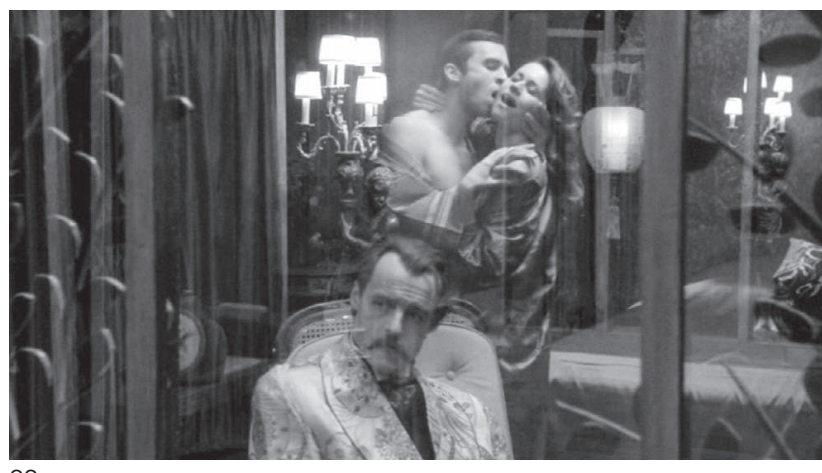

22

mement stylisée: Klimt, d'abord montré à travers le filtre flou d'un plan anamorphosé et nourri de reflets, apparaît ensuite à l'envers, dans le miroir horizontal d'une table qui projette son corps sur les motifs dorés et saturés de ses propres toiles (fig. 25). De semblables effets abstraits s'emparent peu à peu du contrechamp sur Midi : si son visage se découpe d'abord sur un rideau diffusant à contrejour une lumière jaune (fig. 26), le plan suivant la détache sur un fond d'or purement pictural (fig. 27), et le prochain ajoute en surimpression des motifs décoratifs klimtiens qui viennent littéralement dissoudre l'image (fig. 28). Or ces motifs sont exactement les mêmes que ceux qui enveloppent la silhouette féminine du Portrait d'Adèle Bloch-Bauer peint par Klimt en 1907 (fig. 29), l'actrice étant d'ailleurs vêtue et décalée sur la droite de la même façon que l'effigie du tableau. Et si l'on est attentif, on découvre des motifs concordants dans les formes qui entourent le reflet de Klimt sur la table, on se rend même compte que c'est exactement ce Portrait qui trône derrière le peintre, incliné, recadré et inversé dans le miroir, mais bien identique, comme le prouvent la gorge et le bras blancs qui s'y découpent. Raoul Ruiz développe ainsi une sorte de tableau vivant qui se réalise moins par la pose des figures que par la construction de l'image filmique, dont les vertiges perceptifs répondent très exactement aux œuvres de Klimt.

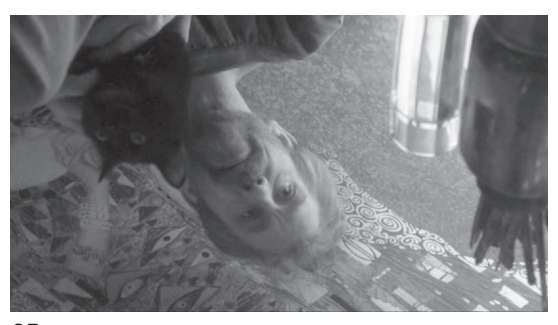

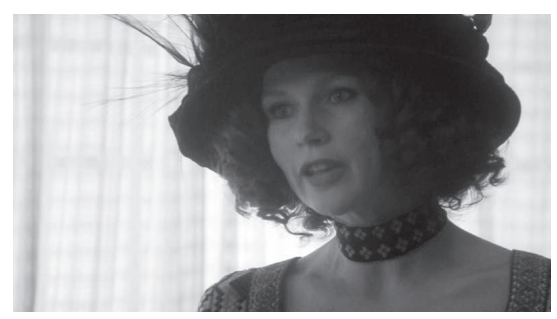

26

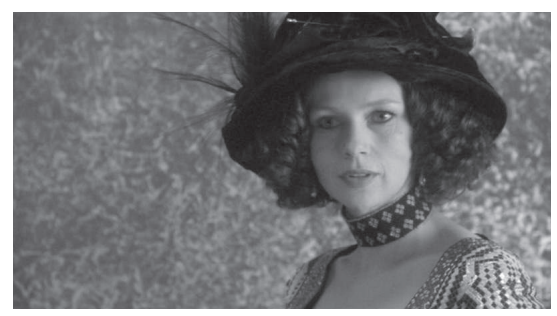

27

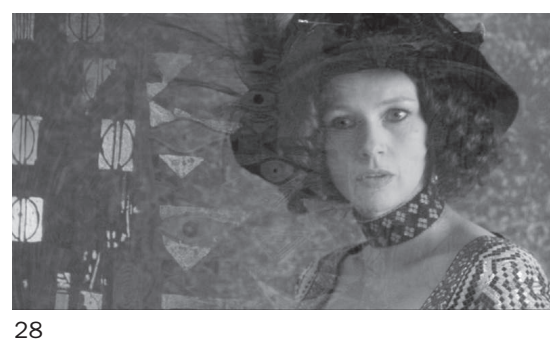




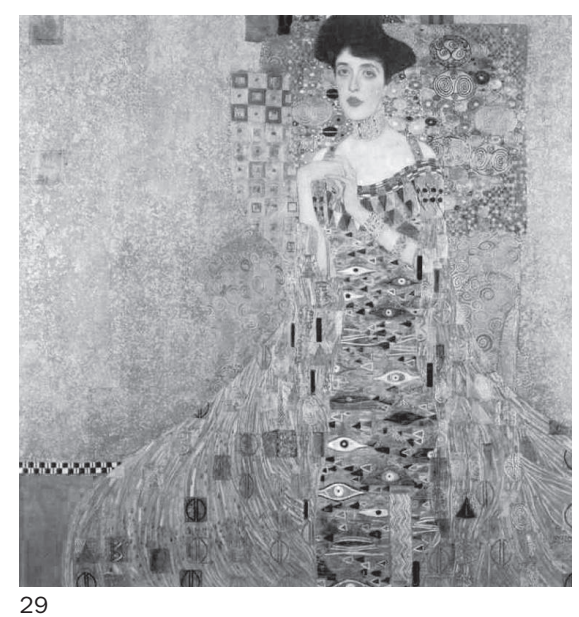

De fait, les tableaux de Gustav Klimt ne traitent pas l'espace de la même manière que les compositions pompières qui ont inspiré Ruiz dans L'Hypothèse du tableau volé et Généalogies d'un crime, loin s'en faut. L'intérêt fondamental du style de Klimt, éminemment manifeste dans Le Baiser, le Portrait d'Adèle Bloch-Bauer et l'ensemble de sa période "dorée», est d'allier un souci naturaliste (ici dans le rendu des corps et des visages) à une stylisation par l'ornement et le fond d'or qui vient effacer toute profondeur au profit de la création d'un espace abstrait et fantasmatique. Cette rupture des règles de la perspective est assurément l'un des aspects de l'œuvre du peintre qui a le plus intéressé Raoul Ruiz. Le cinéaste, extrêmement versé en mathématiques, a en effet souvent expliqué vouloir explorer ses images dans différentes dimensions, en utilisant le cinéma comme une géométrie descriptive :

" $\mathrm{Si}$ on accepte que chaque figure soit réductible à un ensemble de points - chaque point étant à une distance particulière (unique) des autres -, et que de cet ensemble on puisse en "décliner» une figure

38 Raoul Ruiz, Poétique du cinéma 1, op. cit., p. 22.

39 Cf. Le Tableau ou la vision de Dieu (1453), cité par Ruiz dans Poétique du cinéma 2, op. cit., p. 30.

40 Cité par Ruiz dans Pascal Bonitzer et Serge Toubiana, "Entretien avec Raoul Ruiz", op. cit., p. 23.

41 ld., p. 22.

42 Cité par Ruiz dans Poétique du cinéma 1, op. cit., pp. 38-39. en deux, trois, $n$ dimensions, on peut alors aussi accepter qu'ajouter ou enlever des dimensions à une image - sans vraiment la modifier complètement - peut la faire changer de "logique» et donc d'expressivité. » $\mathbf{3 8}$

Raoul Ruiz est fasciné par les théories de la perception qui proposent des conceptions spatiales différentes de celle perspectiviste: le cubisme déjà évoqué, mais aussi l'espace "ultramétrique» décrit par Nicolas de Cues ${ }^{39}$, les «images à six dimensions» de Bertrand Russell 40, la "vision de Dieu» selon Malebranche $\mathbf{4 1}$, le moment de la redécouverte de la vue après une opération de la cataracte décrit par Gaëtan de Clérambault $\mathbf{4 2}$, 
ou encore la «perspective inversée» découverte par Pavel Aleksandro Florenski dans les icônes orthodoxes $\mathbf{4 3}$. L'espace klimtien n'est pas sans actualiser ces conceptions déviantes (et déviées) du monde et des formes qui fascinent Ruiz. Le cinéaste trouve dans les anamorphoses du peintre et sa capacité à instaurer une "incertitude dans la perspective» 44 un "aspect cinématographique» fondamental, comme il s'en explique en comparant certaines modalités de création de l'espace klimtien avec le travail de la caméra :

"[On sait que] Klimt peignait, surtout les paysages, avec un télescope. Dans un documentaire j'ai vu quelque chose d'assez étonnant: quelqu'un a placé la caméra là où se trouvait le télescope. On a mis le même cadre et tout était en place. Il ne manquait que les poules. Mais tout y était, dans un tableau qu'il peignait à peu près à un kilomètre de distance pour chercher cet espèce d'aplatissement qui le fascinait beaucoup, qu'il avait appris à apprécier dans la peinture, surtout byzantine je crois. [...] Cette apparition, cet effet d'aplatissement, c'est une vraie fascination, une émotion cinématographique [...]. » 45

Cet usage d'appareils optiques déformants est exalté par le film qui montre un Klimt moins peintre que "cadreur»46, arpentant les lieux armé, non d'un pinceau, mais d'une petite fenêtre en carton (fig. 30), et fasciné par les miroirs, microscope, théâtre d'ombre, flip book, enfin et surtout par un appareil de projection cinématographique. Raoul Ruiz imagine en effet une rencontre entre Klimt et Méliès : il met en scène l'émerveillement du peintre pour un tel dispositif, et le fait immédiatement fonctionner comme un miroir de celui, pictural et fantasmatique, de Klimt (le peintre se découvre lui-même à l'écran en train de peindre un modèle envoûtant [fig. 31]) $\mathbf{4 7}$. Cette mise en abyme rend explicite la manière dont le film tend à refléter l'univers visuel du peintre. Le tableau vivant du Baiser, noyé dans les reflets de la vitre, décadré en arrière-plan et dédoublé dans la vitre opposée, tout comme la variation sur le Portrait d'Adèle Bloch-Bauer, où l'espace filmique "contaminé» par la toile se déstructure par stylisation, permettent au médium filmique de reconduire la superposition des plans et le défi fait à la perception du spectateur. Le cinéaste, qui se définit comme anti-bazinien, montre ainsi combien l'image cinématographique peut rejouer, dans sa forme, les vertiges visuels des tableaux peints. Et le film tout entier exhibera l'artifice et la construction de ses images en les calquant sur le style saturé de Klimt ${ }^{48}$, permettant au spectateur d'entrer dans l'œil du peintre, d'adopter la même "manière de voir». Le parti pris visuel du film était d'assimiler la caméra au regard du peintre, comme l'explique Raoul Ruiz:
43 ld., pp. 34-35.

44 Hubert Niogret et Philippe Rouyer, "Entretien avec Raoul Ruiz. Dans la tête d'un Klimt improbable", in Positif, n 542, avril 2006, p. 13.

45 "Entretien entre Raoul Ruiz et Serge Lemoine, commissaire de l'exposition Vienne 1900 au Grand Palais ", avril 2006, disponible sur www.lecinemaderaoulruiz.com.

46 Dès la scène d'atelier inaugurale du film, Klimt est montré, non pas en train de peindre ses modèles (qui tiennent une pose déjà spatialement extravagante, pendant du plafond dans des draps), mais en train de préparer son "regard", faisant couler de la vaseline sur une plaque de verre, et l'utilisant comme filtre visuel pour briser la perception traditionnelle et diviser l'image en de multiples facettes et reflets.

47 II n'est pas anodin que ce soit justement Méliès que choisisse Raoul Ruiz, puisque ce prestidigitateur est le premier à avoir utilisé le cinéma comme un trompe-l'œil jouant d'effets, de trucages et d'ornements.

48 Ruiz déploie tout au long du film une esthétique baroque quasi hypnotisante, enchâssant d'incessants mouvements de caméra, faisant tourbillonner le décor autour des personnages, changeant les lumières, déclinant les couleurs, travaillant la surimpression, accumulant les angles de vues multiples et inhabituels, jouant incessamment de surcadrages et de "filtres" qui vont jusqu'à anamorphoser les plans, enfin usant jusqu'à l'excès du motif du miroir, en tous sens et sous toutes ses formes (d'une pièce, en enfilade, sans tain ou brisé en mille morceaux et reflets).

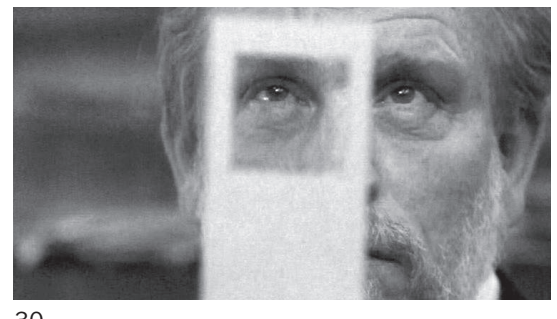


$\mathbf{4 9}$ Hubert Niogret et Philippe Rouyer, "Entretien avec Raoul Ruiz...", op. cit., p. 13.

50 On voit cette situation-cadre au début du film, puis on y revient ponctuellement par des transitions visuelles et sonores (ainsi peut-on soudain entendre, "par-dessus" les images du rêve, la respiration mourante du peintre, ou le clapotis de l'eau dans laquelle son corps malade repose).

51 Op. cit.., p. 11.

52 On peut rapprocher cela d'un autre film qui use du même procédé et choisit aussi le point de vue d'un peintre mourant pour travailler la stylisation onirique et mêler les espaces dans un flou visuel et temporel complet: Stay (Marc Forster, Etats-Unis, 2003).

53 Op. cit.., p. 13.

54 Notons qu'il existe deux versions du film Klimt. Celle qui est éditée en DVD, et sortie à l'origine dans les salles berlinoises et autrichiennes, se présente déjà sur un mode "associatif " très fort, structuré par "décors" dont chacun est un dispositif de regard: l'atelier de Klimt, le restaurant des peintres, la salle de projection filmique, la chambre aux miroirs sans tain, le Klimtmuseum, la "cage" d'un bordel, l'atelier d'un photographe, le studio et théâtre d'ombres de Méliès, etc. Or cette version est le fruit d'un remontage, demandé par le principal producteur autrichien, de la version initiale - director's cut - du film, qui désormais semble inaccessible (mais qui a été projetée dans les salles françaises en avril-mai 2006). Et si l'on en croit Antoine de Baecque, la version originale était bien plus fantasmatique et bien moins linéaire encore, celui-ci assurant que le remontage, qu'il taxe de "charcutage pervers", aurait "passé à la moulinette la plupart de ce qui fait l'intérêt de ce film ses divagations, fantasmagories, répétitions, résurgences, jeux de renvois et de miroirs, ses dédoublements et ses escamotages". (Antoine de Baecque, "Klimt cousu d'or. Une fantaisie de Ruiz, comme rêvée par le peintre", www. libération.fr, 26 avril 2006).

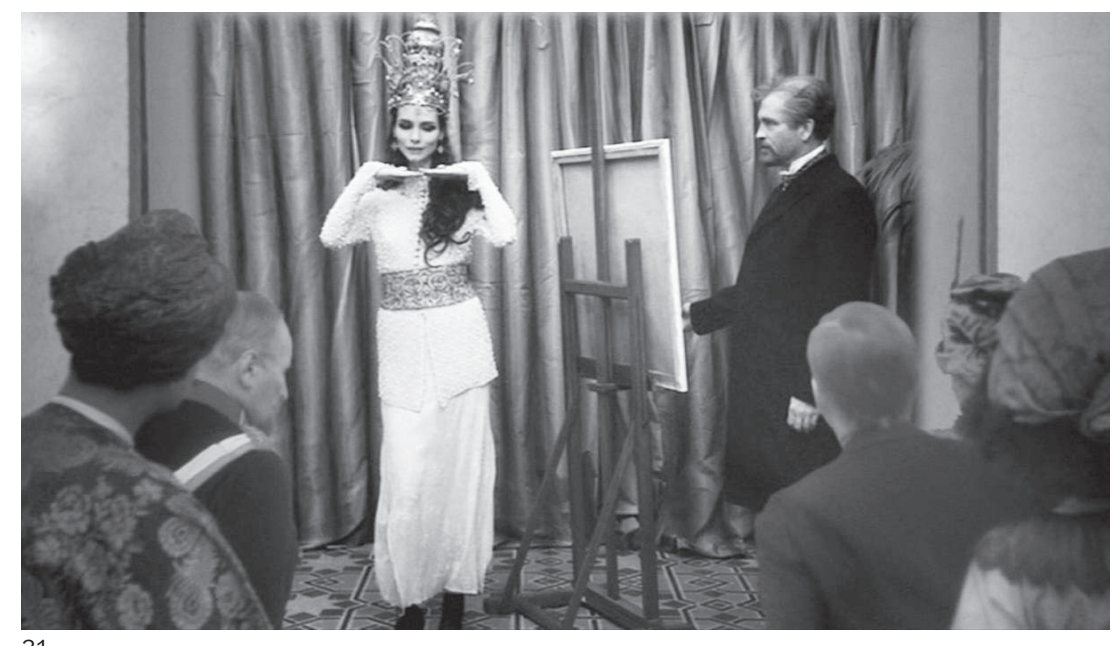

31

«Pour moi c'était un peu comme me mettre dans la tête d'un Klimt improbable qui aurait eu la possibilité de tourner des films. »49

Comme l'annonce cette citation (où il s'agit d'entrer, non pas seulement dans les yeux, mais bien "dans la tête» du peintre), l'expérience perceptive dépasse le caractère purement visuel. En effet, l'ensemble du film se présente comme un rêve vécu en focalisation interne par un Klimt délirant, en train de mourir sur son lit d'hôpital50. Cela donne aux images du film un degré d'artifice d'autant plus légitime:

"Comme on dit souvent, au moment de mourir il revoit toute sa vie. Sauf que lui, la vie qu'il revoit n'est pas forcément celle qu'il a vécue. C'est une vie à côté, une vie en puissance qui reprend tous les éléments de sa vraie vie, mais différemment. » $\mathbf{5 1}$

C'est donc une sorte de «near death experience» (ou «expérience de mort imminente») à laquelle nous convie Ruiz, expérimentant dans leurs derniers retranchements les visions oniriques et stylisées d'un peintre dont l'ensemble de l'œuvre travaille les abîmes du regard $\mathbf{5 2}$. Toute linéarité temporelle est court-circuitée au profit d'une logique purement visuelle:

"Le scénario nous dispensait de nous soucier de la chronologie [...] puisque dans sa tête tout se mélange. [...] C'est par les associations d'images que la majeure partie du film fonctionne. »53

Le film mime ainsi le fonctionnement de la perception inconsciente, onirique et automatique ${ }^{54}$. Raoul Ruiz est en effet extrêmement intéressé par la perception mentale inconsciente; et s'il tente de la représenter dans Klimt, il a toujours prôné l'automatisme onirique comme paradigme pour la structure de ses films et pour leur réception elle-même. 
Ses œuvres cherchent à activer ce type de pensée, aux antipodes de la déduction logique et linéaire sollicitée par les films classiques, quitte à ce que le spectateur s'endorme et «rêve» pendant et par-dessus le film, ou quitte à ce que chacun ait une perception différente des images $\mathbf{5 5}$. Ruiz a précisé vouloir solliciter dans la perception du spectateur une mémoire périphérique, implicite et non localisée, appelée le "priming", qui est essentiellement émotionnelle et déclenche les réflexes mémoriels, les associations d'idées, les rêves, etc. 56 Pour ce faire, l'une des tactiques travaillées dans Klimt consiste à utiliser l'arrière-plan :

"En général, la production hollywoodienne cherche à faire converger l'information sur les protagonistes, sur ce qui est devant. Tout ce qui est à l'arrière-plan est pour ainsi dire inerte. Parce qu'on part du principe que, au cinéma, on n'a pas le temps d'analyser le détail d'une image. On peut néanmoins le percevoir, et un certain nombre de cinéastes ont travaillé sur ce principe intuitivement. [...] A quel moment peut-on avoir la certitude qu'un élément expressif a été perçu ? Jusqu'à quel point faut-il le mettre en évidence? Ce qui fait la richesse des films, c'est bien sûr ce type de mémoire involontaire.» $\mathbf{5 7}$ Or, le tableau vivant du film Klimt est le lieu le plus emblématique de cette exploration de l'arrière-plan, et ses "avatars" (les modèles tenant la pose des Amies, les indices pour reconnaître le Portrait d'Adèle BlochBauer) sont tous logés dans cette zone floue, sollicitant une perception inconsciente, plongeant dans la mémoire périphérique. Ainsi, encore une fois, Ruiz utilise le tableau vivant comme moyen par excellence d'expérimenter la perception, qui est le véritable sujet de Klimt, et, à mes yeux, du cinéma de Raoul Ruiz $\mathbf{5 8}$.

\section{Le tableau vivant ou chaque aspect d'un instant}

Le tableau vivant constitue donc un motif d'analyse privilégié de l'œuvre de Raoul Ruiz: en tant que dispositif de regard, il reflète les expérimentations du cinéaste sur la perception du spectateur, sa volonté d'explorer la vision au-delà de tout cadre, tant visuel que conceptuel. Or, dans la filmographie du cinéaste chilien - elle-même inépuisable et forçant à la spéculation en raison de la quantité de films inaccessibles nombreux sont les motifs comparables au tableau vivant, actualisant une semblable mise en "pose» de la temporalité au profit d'une même libération de la perception. On peut ainsi citer le principe du court métrage Colloque de chien (France, 1977), qui ne travaille pas autour de tableaux vivants mais est structuré par des «arrêts sur image». Cette suite d'«instants prégnants» permet de rendre visible l'engrenage du récit qui se noue en cercles vicieux. On peut également évoquer les poses
55 Voir Poétique du cinéma 1, op. cit., p. 39. Notons qu'à la base de ces expériences réside ce que Ruiz nomme la "superstition (ou croyance, ou vérité scientifique soutenue par l'expérience)" selon laquelle "le cinéma serait l'art d'exciter une partie du cerveau qui ne fonctionne que pendant le sommeil en la bombardant d'images statiques juxtaposées afin de créer l'illusion du mouvement (tandis que la vidéo, dont l'image est liquide, exciterait une autre partie, opérationnelle seulement lorsque le corps est éveillé). "(Ibid.).

56 Ruiz s'est souvent exprimé sur la découverte par des neurologues tels que Daniel Schacter de ces "morceaux de mémoire dispersés dans le cerveau et activés grâce à l'émotion", et sur sa volonté de "s'en servir dans les films". Voir Hubert Niogret et Philippe Rouyer, "Entretien avec Raoul Ruiz..." op. cit., pp. 13-14, ou «Entretien avec Pierre-Alexandre Nicaise et Eric Lebot, 9 mars 2007 ", www. lecinemaderaoulruiz.com, p. 3.

57 Hubert Niogret et Philippe Rouyer, id., p. 14.

58 Afin de montrer cette importance de la question du point de vue on peut se borner à citer, dans la filmographie du cinéaste, les titres suivants:

- Le Borgne (France, 1980), qui fonctionne en caméra subjective dans la perception d'un homme mort;

- Les Trois Couronnes du matelot (France/ Chili, 1982), qui s'inspire des effets travaillés en BD par Milton Caniff pour étrangéifier la vision en s'interdisant de faire plusieurs plans à partir du même axe de vue;

- Point de fuite (France, 1984);

- Dans un miroir (France, 1984);

- Voyage d'une main (France, 1984, annoncé d'abord sous le titre Voyage autour de ma chambre, et présenté comme une expérience de vision omnidirectionnelle et divine avec un fétichisme du motif de l'œil et du trucage optique, comme en témoigne le storyboard publié dans les Cahiers du cinéma, n³ 345, mars 1983, pp. 55-56, dessiné par Karsiu Lee, le peintre des tableaux de L'Hypothèse du tableau volé;

- Mémoire des apparences (France, 1986, qui met en scène une tentative de mémorisation;

- La Chouette aveugle (France/Suisse, 1987), qui dépeint les jeux de regards entre un projectionniste et l'héroïne du film qu'il montre;

- L'CEil qui ment (France/Portugal, 1991), qui travaille autour des visions miraculeuses;

- Miotte vu par Ruiz (France/Suisse, 2001), qui fait voir la création picturale de l'artiste. 
59 Le freeze est un phénomène social qui se popularise depuis la fin des années 2000; il consiste en un rassemblement de personnes qui, dans un lieu donné (via un rendez-vous par internet), se figent toutes ensemble en plein mouvement, pendant plusieurs minutes sous le regard interloqué des passants. Ces actions surnommées freeze-party (qui, pour la plupart, fonctionnent comme un pur divertissement) réactualisent le saisissement provoqué par l'immobilisation (d'autant plus impressionnante en pleine agitation urbaine) qui participait grandement à la séduction du tableau vivant au XIXe siècle.

60 Raoul Ruiz, "A propos du Temps retrouvé (propos recueillis par Jacinto Lageira et Gilles A. Tiberghien, mars 1999)", dans Raoul Ruiz. Entretiens, op. cit., p. 78.

$61 \mathrm{lbid}$.

62 ld., p. 79.

63 lbid.

64 A l'instar de son court métrage sur Dali (Pages d'un catalogue, France, 1980), où il matérialise l'espace d'une toile dans une tridimensionnalité illusionniste d'où surgissent des "bras" encombrés d'objets surréalistes, ou de son Petit manuel d'histoire de France (France, 1979) qui semble, comme le laisse penser une photographie du film publiée dans les Cahiers du cinéma, $\mathrm{n}^{\circ} 345,1983$, p. 17, reconstituer les tableaux qui façonnent la manière de percevoir I'histoire.

65 "Avec [Trois vies et une seule mort], je faisais l'expérience d'une structure cubiste où tout se superposait. Mais je ne connaissais pas [les philosophes du miroir, qui sont en fait des physiciens: David Bohm, Karl Pribram], je ne les ai lus qu'après Proust." (Hubert Niogret et Philippe Rouyer, "Entretien avec Raoul Ruiz...", op. cit., p. 13).

66 Réplique du personnage joué par Marcello Mastroianni dans Trois vies et une seule mort, $21^{\mathrm{e}}$ minute. qui surviennent dans Le Temps retrouvé (France, 1999): les passants qui occupent l'arrière-plan sont tout à coup "arrêtés " comme dans un freeze contemporain 59 ; soudain, le héros trébuche et reste figé dans cette position "suspendue» tandis que l'espace autour de lui change et que les mouvements s'y accélèrent... Ruiz explique de fait avoir filmé la narration proustienne «en tant que tableau» $\mathbf{6 0}$, afin de donner une forme visuelle aux digressions de La Recherche du temps perdu, où « une situation renvoie à une autre» $\mathbf{6 1}$ suivant la dynamique cyclique de la répétition "qui nous conduit sans cesse au point de départ» 62. C'est donc encore une fois une manière d'explorer la perception et ses retranchements cubistes où plusieurs scènes et espaces-temps peuvent se superposer, qui a motivé cette esthétique de "l'image sans mouvement»63. Ces jeux sont d'ailleurs annoncés tout au début du film dans une séquence "prégnante» où l'on découvre Marcel, enfant, qui face au public de la société use d'une lanterne magique pour projeter des images kaléidoscopiques sur les murs, mais ceux-ci «s'effacent», l'espace s'étend, et les images prennent corps sur des modèles vivants immobiles, grimés en sculptures vivantes. Bien d'autres "variations" autour du tableau vivant pourront encore être trouvées dans les films passés et futurs de Raoul Ruiz $^{64}$; nous nous contenterons, pour finir, d'évoquer celle qui survient dans Trois Vies et une seule mort (France, 1996). Les mots pour décrire ce motif, que Raoul Ruiz a prêté au héros de cette fiction - qu'il a luimême définie comme «cubiste» $\mathbf{6 5}$ - nous permettront de conclure que mettre une scène «en pose» est pour Ruiz un moyen incomparable de la contempler, en réveillant et en décuplant les facultés perceptives du spectateur. Incarné par Marcello Mastroianni, le personnage de ce film raconte à un passant (Feodor Atkine) comment les fées se sont jouées de lui. Or l'un de leurs tours peut être rigoureusement assimilé à celui que le cinéaste Raoul Ruiz nous joue à nous, spectateurs, lorsqu'il nous confronte à des tableaux vivants :

«Cette fois-là [les fées] m'avaient préparé une autre plaisanterie. Il ne s'agissait plus de faire défiler [le temps]: pendant des mois je fus condamné à rester prisonnier d'un seul instant. J'ai eu le loisir d'examiner chaque aspect d'un instant, et c'est plus compliqué que ce qu'on croit... » 66 